\title{
Atomic effective potentials for starting molecular electronic structure calculations
}

\author{
Dimitri N. Laikov • Ksenia R. Briling
}

the date of receipt and acceptance should be inserted later

\begin{abstract}
Atomic effective one-electron potentials in a compact analytic form in terms of a few Gaussian charge distributions are developed, for Hydrogen through Nobelium, for starting molecular electronic structure calculations by a simple diagonalization. For each element, all terms but one are optimized in an isolated-atom Hartree-Fock calculation, and the last one is parametrized on a set of molecules. This one-parameter-per-atom model gives a good starting guess for typical molecules and may be of interest even on its own.
\end{abstract}

\section{Introduction}

Every molecular electronic structure calculation needs a good starting guess. Most likely the earliest and still widely used extended Hückel [1] theory gives a minimalbasis solution that can be then projected [2] onto the working basis set. The underlying minimal basis should be given in some way — some workers [3] used short three-Gaussians-per-shell sets [4] for atoms up to Radon, with known shortcomings. We thought it would be handy to have a tool to build a better minimal set straight from the working set - clearly, atomic Hartree-Fock calculations could be done for that, but even then a starting guess is needed. We have found that the optimized effective potentials [5, 6] for atoms can be given, accurately enough, in a very compact form in terms of Gaussian charge distributions - they can be pre-tabulated and used to get the starting guess for an atom with one diagonalization of an easily-computable matrix. One may wonder whether this has already been done for all elements, but we see only works of this kind 7 with rather complicated, and even piecewise, analytic form of the potential.

D. N. Laikov

Chemistry Department, Moscow State University, 119991 Moscow, Russia

E-mail: laikov@rad.chem.msu.ru

K. R. Briling

Chemistry Department, Moscow State University, 119991 Moscow, Russia

E-mail: briling@rad.chem.msu.ru 
Another well-known starting guess for molecules uses an effective one-electron Hamiltonian of density-functional theory [8] within the approximation [9] of overlapped but frozen atomic densities. The nonlinearity of the exchange-correlation functionals makes the matrix elements of the potential tractable only by numerical integration, for which good schemes [10,11] are known but need to be implemented. A linearized version thereof is simply the sum of fixed neutral-atom potentials taken from a density-functional calculation, which, remarkably, has been introduced and benchmarked for the first time only a few months ago [12]. There is also a Hartree-Fock analog [13] that needs up to three-center two-electron integrals, or its spherical approximation [14] with less costly models of the direct and exchange terms.

Of course, the idea to approximate the molecular effective one-electron potential by a sum of atom-centered functions is not new and dates back to the early density-functional works [15], where the Coulomb part was calculated using density fitting and the exchange-correlation potential was also fitted, either on the grid [16], or, in the special case of Hartree-Fock-Slater [17] theory, by an elegant grid-free variational method [18] with analytical evaluation of all three-center integrals. Though the approximation of both Coulomb and exchange-correlation terms by an electrostatic potential arising from a sum of atom-centered (Gaussian-type) effective charge distributions was put forward by one of us [19] more than 20 years ago, there seems to be a new interest in using this functional form to derive transferable atomic potentials for molecules in educational work [20] and beyond [21, 22 .

Our atom-optimized compact representations can also be adopted and adapted for molecules by "capping" each atom with one more adjustable potential term to cancel the $-1 / r$ asymptotic behavior and make it short-ranged, this way we get a new tunable model worth being studied. Our tests show it to be at least as good as an extended Hückel guess, we also compare it with the sum of tailless Hartree-Fock-Slater-based neutral-atom potentials and we see that our tunable model often works much better, so we report it here in the hope that it may be helpful for others.

\section{Theory}

For an atom, our effective potential $v(\mathbf{r})$ is the sum of its bare-nuclear potential $v_{\mathrm{n}}(\mathbf{r})$ and an approximate (direct and exchange) screening term

$$
v_{\mathrm{a}}(\mathbf{r})=\sum_{i=1}^{n} c_{i} \frac{\operatorname{erf}\left(\sqrt{a_{i}}|\mathbf{r}|\right)}{|\mathbf{r}|}
$$

in the form of Coulomb potential of Gaussian [23] charge distributions. We optimize the exponents $a_{i}$ and coefficients $c_{i}$, with the constraint

$$
\sum_{i=1}^{n} c_{i}=Q-1
$$

$Q$ being the nuclear charge, to minimize the measure

$$
\mathcal{E}=\frac{1}{2} \iint \frac{\left(\rho_{v}\left(\mathbf{r}_{1}\right)-\rho_{0}\left(\mathbf{r}_{1}\right)\right)\left(\rho_{v}\left(\mathbf{r}_{2}\right)-\rho_{0}\left(\mathbf{r}_{2}\right)\right)}{\left|\mathbf{r}_{1}-\mathbf{r}_{2}\right|} \mathrm{d}^{3} \mathbf{r}_{1} \mathrm{~d}^{3} \mathbf{r}_{2}
$$


Table 1 Atomic parameters.

\begin{tabular}{lrrcc}
\hline Atoms & $n$ & $a_{n+1}$ & $\mathcal{E}^{\mathrm{a}}$ & $\Delta E^{\mathrm{a}, \mathrm{b}}$ \\
\hline $\mathrm{H}$ & 0 & $1 / 3$ & & \\
$\mathrm{He}$ & 1 & $1 / 3$ & 0.000024 & 0.0004 \\
$\mathrm{Li}$ & 1 & $1 / 16$ & 0.000154 & 0.0050 \\
$\mathrm{Be}$ & 2 & $1 / 16$ & 0.000075 & 0.0040 \\
$\mathrm{~B}-\mathrm{Ne}$ & 2 & $1 / 3$ & 0.000320 & 0.0094 \\
$\mathrm{Na}$ & 2 & $1 / 32$ & 0.000379 & 0.0135 \\
$\mathrm{Mg}$ & 3 & $1 / 32$ & 0.000099 & 0.0069 \\
$\mathrm{Al}-\mathrm{Ar}$ & 3 & $1 / 8$ & 0.000143 & 0.0103 \\
$\mathrm{~K}-\mathrm{Ca}$ & 3 & $1 / 32$ & 0.000275 & 0.0138 \\
$\mathrm{Sc}-\mathrm{Zn}$ & 4 & $1 / 6$ & 0.000029 & 0.0191 \\
$\mathrm{Ga}-\mathrm{Kr}$ & 4 & $1 / 12$ & 0.000180 & 0.0210 \\
$\mathrm{Rb}-\mathrm{Sr}$ & 4 & $1 / 32$ & 0.000085 & 0.0159 \\
$\mathrm{Y}-\mathrm{Cd}$ & 4 & $1 / 8$ & 0.000391 & 0.0326 \\
$\mathrm{In}-\mathrm{Xe}$ & 5 & $1 / 12$ & 0.000035 & 0.0225 \\
$\mathrm{Cs}-\mathrm{Yb}$ & 5 & $1 / 32$ & 0.000205 & 0.0519 \\
$\mathrm{Lu}-\mathrm{Hg}$ & 5 & $1 / 12$ & 0.000239 & 0.0537 \\
$\mathrm{Tl}-\mathrm{Rn}$ & 6 & $1 / 12$ & 0.000069 & 0.0450 \\
$\mathrm{Fr}-\mathrm{No}$ & 6 & $1 / 32$ & 0.000297 & 0.0835 \\
\hline
\end{tabular}

a The largest value in the row.

b Energy error.

of the difference between the Hartree-Fock $\rho_{0}$ and the effective-potential-based $\rho_{v}$ densities. Spherically-averaged configurations [24] are taken for atoms with open-shell ground states. Eq. (2) ensures the right asymptotic behavior of $v(\mathbf{r})$ as $|\mathbf{r}| \rightarrow \infty$. We use the four-component scalar-relativistic [25] Hamiltonian and a finite Gaussian [26] nucleus model for all atoms Hydrogen through Nobelium.

Table 1 shows the number of terms $n$ and the value of $\mathcal{E}$ for the groups of atoms, the overall accuracy is quite good, $\mathcal{E}<0.0004 ; n-1$ terms would raise $\mathcal{E}$ by about 10 times, while $n+1$ can hardly do any better and would often lead to runaway solutions. We also check the atomic Hartree-Fock energy errors $\Delta E$ within a two-component scalar-relativistic approximation [27] thanks to its boundedness from below.

For a molecule with $N$ atoms, the atomic potential tails of $-1 / r$ would have summed up to an unphysical $-N / r$; as a lesser evil, we decided to "cap" each atom with one more term having $c_{n+1}=1$ and a to-be-optimized $a_{n+1}$. (Setting $c_{n+1}=(N-1) / N$ may be kept as an option.) This also gives us the freedom to adjust the potential well depth in the valence region to mimic a typical molecular environment.

For the optimization, we tried two objective functions: (a) the energy-based

$$
f_{\mathrm{E}}=\sum_{i}\left(\left\langle\phi_{i}\left|\hat{F}^{0}\right| \phi_{i}\right\rangle-\left\langle\phi_{i}^{0}\left|\hat{F}^{0}\right| \phi_{i}^{0}\right\rangle\right)
$$

or (b) the overlap-based

$$
f_{\mathrm{S}}=\sum_{j}\left(1-\sum_{i}\left|\left\langle\phi_{i} \mid \phi_{j}^{0}\right\rangle\right|^{2}\right)
$$


where the wavefunctions $\phi_{j}^{0}$ and the Fock operator $\hat{F}^{0}$ are taken from the reference self-consistent field calculation, whereas $\phi_{i}$ are our approximate solutions, the (spin and space) labels $i$ and $j$ run over the occupied valence set. The functions, either $f_{\mathrm{E}}$ or $f_{\mathrm{S}}$, are to be summed up over a training set of molecules and minimized with respect to the atomic parameters.

Our thus derived potentials can work equally well with any all-electron formalism, both two- and four-component. They can also be easily adapted for use with effective core potentials [28] by subtracting the core charge $Q_{0}$ from $c_{i}$ referring to the largest $a_{i}$,

$$
\bar{c}_{i}=\left\{\begin{array}{cr}
0, & i<\bar{n}, \\
\sum_{j=1}^{\bar{n}} c_{j}-Q_{0}, & i=\bar{n}, \\
c_{i}, & i>\bar{n},
\end{array}\right.
$$

with $\bar{n}$ such that $0 \leq \bar{c}_{i} \leq c_{i}$, where $a_{i}>a_{i+1}$ for all $i$.

\section{Calculations}

The problem of Eq. (3) was solved by a code written by one of us (DNL) within the atomic structure program 24, while the further optimization of parameters on molecules was done by an original program 11 written by the other (KRB), and then the starting guess for molecules was double-checked by each of us independently by one's own molecular electronic structure code.

The Hartree-Fock-optimized atomic parameters for Helium through Nobelium are tabulated in the Supplementary material. The reference molecular calculations were done with the PBE [29] density functional, the scalar-relativistic approximation [27, and the L1 basis [30,24].

First, we optimized the $a_{n+1}$ parameters for atoms $\mathrm{H}$ through $\mathrm{Cl}$ (without $\mathrm{He}$ and $\mathrm{Ne}$ ) on the neutral closed-shell subset of molecules [31. For $\mathrm{H}$ and $\mathrm{B}-\mathrm{F}$ we got the values strikingly close to $1 / 3$ - this, together with the flatness near the minimum, led us to have all parameter values within groups of atoms constrained to be equal during the optimization and rounded afterwards to simple fractions. For the heavier elements, skipping $\mathrm{Pr}-\mathrm{Tm}$ and $\mathrm{Np}-\mathrm{Md}$ with their open-shell states, we have built up a smaller set of prototypical molecules and gotten the rest of values shown in Table 1 . On average, we get the energy error per atom $f_{E} / N \approx 0.005$ au for the lighter, and $\approx 0.009$ au for the heavier, subsets.

Taking atomic Hartree-Fock-Slater densities as $\rho_{0}$ in Eq. (3) and setting the constraint with $Q$ on the right hand side of Eq. (2), we have optimized another set of potentials of Eq. (1) now with $n$ simply equal to the highest occupied principal quantum number in the atom, they are also tabulated in the Supplementary material for all 102 elements. Symptomatically, we get $a_{1} \approx 0.08$ for Hydrogen, rather diffuse compared to our optimized value of $1 / 3$. On our set of molecules, we get systematically higher error measures $f_{E}$ in most cases when the parametrized potentials are replaced with their Hartree-Fock-Slater-based analogs, typically by $\sim 10$ times for the organic subset (a full listing can be found in the Supplementary material), thus our parametrization is indeed helpful.

\footnotetext{
1 To be found at https://github.com/briling/aepm
} 


\section{Conclusions}

It would be straightforward to implement our starting guess, as an option, into any electronic structure code - for Gaussian basis sets, all the integrals can be easily computed analytically [23, 32. On the other hand, this elegant one-parameter-peratom model may be of interest even on its own. Followed by an energy correction in the spirit of second-order perturbation theory [33, 34, 35, 36, it may try to replace the iterative self-consistent solution in lower-accuracy applications.

An unnamed reader has warned us of the dangers of black boxes [37, and though we find our potentials to work well in the raised case of ferrocene, one should always keep in mind that a guess is a guess, however good it may be on average.

\section{Supplementary material}

File format description (readme.txt), atomic parameters based on Hartree-Fock (ac.txt) and Hartree-Fock-Slater (ac0.txt) theory, and error measures for all molecules (fm.txt).

\section{References}

1. R. Hoffmann, J. Chem. Phys. 39, 1397 (1963). DOI 10.1063/1.1734456

2. H.F. King, R.E. Stanton, H. Kim, R.E. Wyatt, R.G. Parr, J. Chem. Phys. 47, 1936 (1967). DOI 10.1063/1.1712221

3. M.W. Schmidt, K.K. Baldridge, J.A. Boatz, S.T. Elbert, M.S. Gordon, J.H. Jensen, S. Koseki, N. Matsunaga, K.A. Nguyen, S. Su, T.L. Windus, M. Dupuis, J.A. Montgomery, J. Comput. Chem. 14, 1347 (1993). DOI 10.1002/jcc.540141112

4. S. Huzinaga, J. Andzelm, M. Klobukowski, E. Radzio-Andzelm, Y. Sakai, H. Tatewaki, Gaussian basis sets for molecular calculations, Physical sciences data, vol. 16 (Elsevier, Amsterdam, 1984)

5. R.T. Sharp, G.K. Horton, Phys. Rev. 90, 317 (1953). DOI 10.1103/physrev.90.317

6. J.D. Talman, W.F. Shadwick, Phys. Rev. A 14, 36 (1976). DOI 10.1103/physreva.14.36

7. P. Maldonado, A. Sarsa, E. Buendía, F. Gálvez, Atom. Data Nucl. Data 97, 109 (2011). DOI 10.1016/j.adt.2010.10.002

8. W. Kohn, L.J. Sham, Phys. Rev. 140, A1133 (1965). DOI 10.1103/PhysRev.140.A1133

9. J. Harris, Phys. Rev. B 31, 1770 (1985). DOI 10.1103/physrevb.31.1770

10. A.D. Becke, J. Chem. Phys. 88, 2547 (1988). DOI 10.1063/1.454033

11. H. Laqua, J. Kussmann, C. Ochsenfeld, J. Chem. Phys. 149, 204111 (2018). DOI 10.1063/ 1.5049435

12. S. Lehtola, J. Chem. Theory Comput. 15, 1593 (2019). DOI 10.1021/acs.jctc.8b01089

13. J.H. Van Lenthe, R. Zwaans, H.J.J. Van Dam, M.F. Guest, J. Comput. Chem. 27, 926 (2006). DOI 10.1002/jcc.20393

14. L. Amat, R. Carbó-Dorca, Int. J. Quantum Chem. 87, 59 (2001). DOI 10.1002/qua.10068

15. H. Sambe, R.H. Felton, J. Chem. Phys. 62, 1122 (1975). DOI 10.1063/1.430555

16. B.I. Dunlap, J.W.D. Connolly, J.R. Sabin, J. Chem. Phys. 71, 3396 (1979). DOI 10.1063/ 1.438728

17. J.C. Slater, Phys. Rev. 81, 385 (1951). DOI 10.1103/PhysRev.81.385

18. B.I. Dunlap, J. Phys. Chem. 90, 5524 (1986). DOI 10.1021/j100280a010

19. D.N. Laikov, Chem. Phys. Lett. 281, 151 (1997). DOI 10.1016/S0009-2614(97)01206-2

20. F. Nazari, J.L. Whitten, J. Chem. Phys. 146, 194109 (2017). DOI 10.1063/1.4983395

21. J.L. Whitten, J. Chem. Phys. 150, 034107 (2019). DOI 10.1063/1.5064781

22. J.L. Whitten, Phys. Chem. Chem. Phys. 21, 21541 (2019). DOI 10.1039/c9cp02450f

23. S.F. Boys, Proc. R. Soc. A 200, 542 (1950). DOI 10.1098/rspa.1950.0036

24. D.N. Laikov, Theor. Chem. Acc. 138, 40 (2019). DOI 10.1007/s00214-019-2432-3 
25. K.G. Dyall, J. Chem. Phys. 100, 2118 (1994). DOI 10.1063/1.466508

26. L. Visscher, K.G. Dyall, Atom. Data Nucl. Data 67, 207 (1997). DOI 10.1006/adnd.1997. 0751

27. D.N. Laikov, J. Chem. Phys. 150, 061103 (2019). DOI 10.1063/1.5082231

28. L.R. Kahn, P. Baybutt, D.G. Truhlar, J. Chem. Phys. 65, 3826 (1976). DOI 10.1063/1. 432900

29. J.P. Perdew, K. Burke, M. Ernzerhof, Phys. Rev. Lett. 77, 3865 (1996). DOI 10.1103/ PhysRevLett.77.3865

30. D.N. Laikov, Chem. Phys. Lett. 416, 116 (2005). DOI 10.1016/j.cplett.2005.09.046

31. D.N. Laikov, J. Chem. Phys. 135, 134120 (2011). DOI 10.1063/1.3646498

32. L.E. McMurchie, E.R. Davidson, J. Comput. Phys. 26, 218 (1978). DOI 10.1016/ 0021-9991(78)90092-x

33. S. Havriliak, H.F. King, J. Am. Chem. Soc. 105, 4 (1983). DOI 10.1021/ja00339a002

34. K. Wolinski, P. Pulay, J. Chem. Phys. 118, 9497 (2003). DOI 10.1063/1.1562606

35. J. Deng, A.T.B. Gilbert, P.M.W. Gill, J. Chem. Phys. 130, 231101 (2009). DOI 10.1063/ 1.3152864

36. J. Deng, A.T.B. Gilbert, P.M.W. Gill, J. Chem. Phys. 133, 044116 (2010). DOI 10.1063/ 1.3463800

37. J. Martin, J. Baker, P. Pulay, J. Comput. Chem. 30, 881 (2009). DOI 10.1002/jcc.21106 


$$
\text { file "readme.txt" }
$$

Supplementary material

for

"Atomic effective potentials for starting molecular electronic structure calculations" by

Dimitri N. Laikov and Ksenia R. Briling

The file "ac.txt" holds parameters of atomic effective potentials

for atoms Helium through Nobelium in the format:

the atomic number and the number of terms $n$

followed by $n$ pairs of the exponent and coefficient, one per line.

For example:

\section{3}

$+.70097817629160849215 \mathrm{e}+02+.33299240013050428956 \mathrm{e}+01$

$+.42193314636035713068 \mathrm{e}+01+.77190281538706445101 \mathrm{e}+01$

$+.35198503878294074576 \mathrm{e}+00+.59510478448243125943 \mathrm{e}+01$

for atomic number 18 (Argon) 3 (three) terms are listed

with exponents $\mathrm{a} 1=70.1, \mathrm{a} 2=4.2, \mathrm{a} 3=0.35$,

and coefficients $c 1=3.3, c 2=7.7, c 3=6.0$ (rounded).

The Hartree-Fock-Slater analogs are in the file "ac0.txt", for comparison.

Molecular tests are documented in the file "fm.txt": for each molecule (column 1), the energy (Eq. 4) errors

for parametrized (column 2) and Hartree-Fock-Slater-based (column 3)

potentials are listed followed by the overlap (Eq. 5)

errors (columns 4 and 5), all values in a.u. 


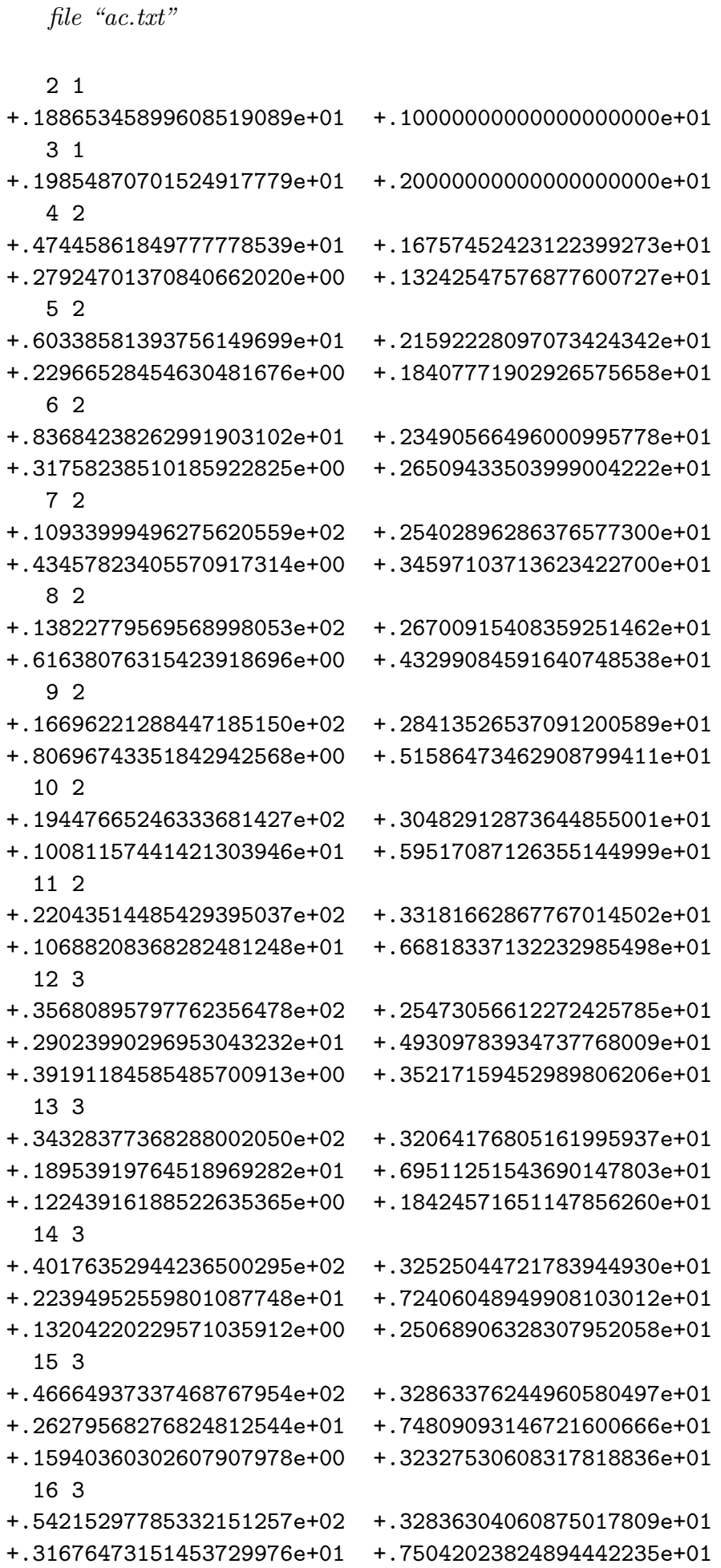




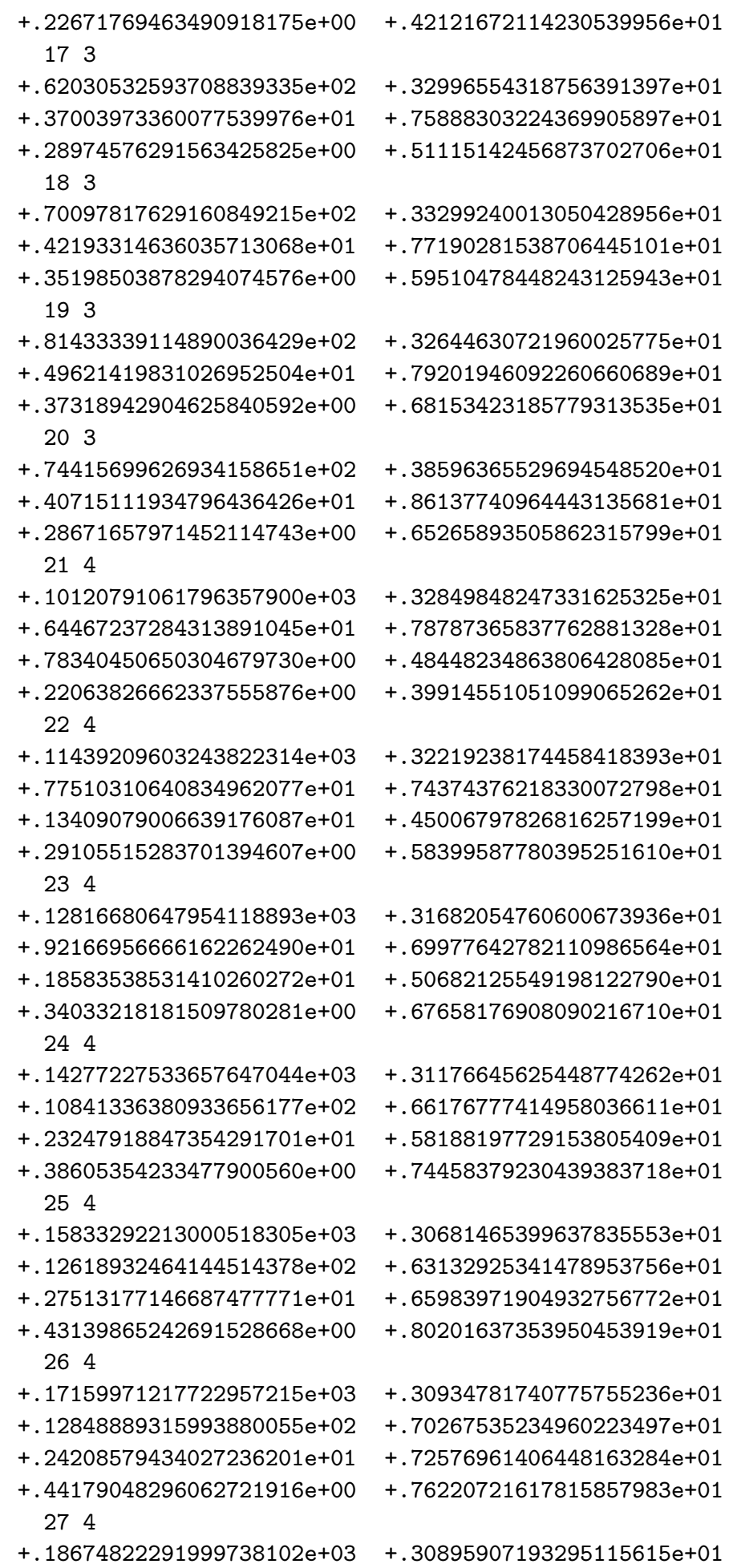




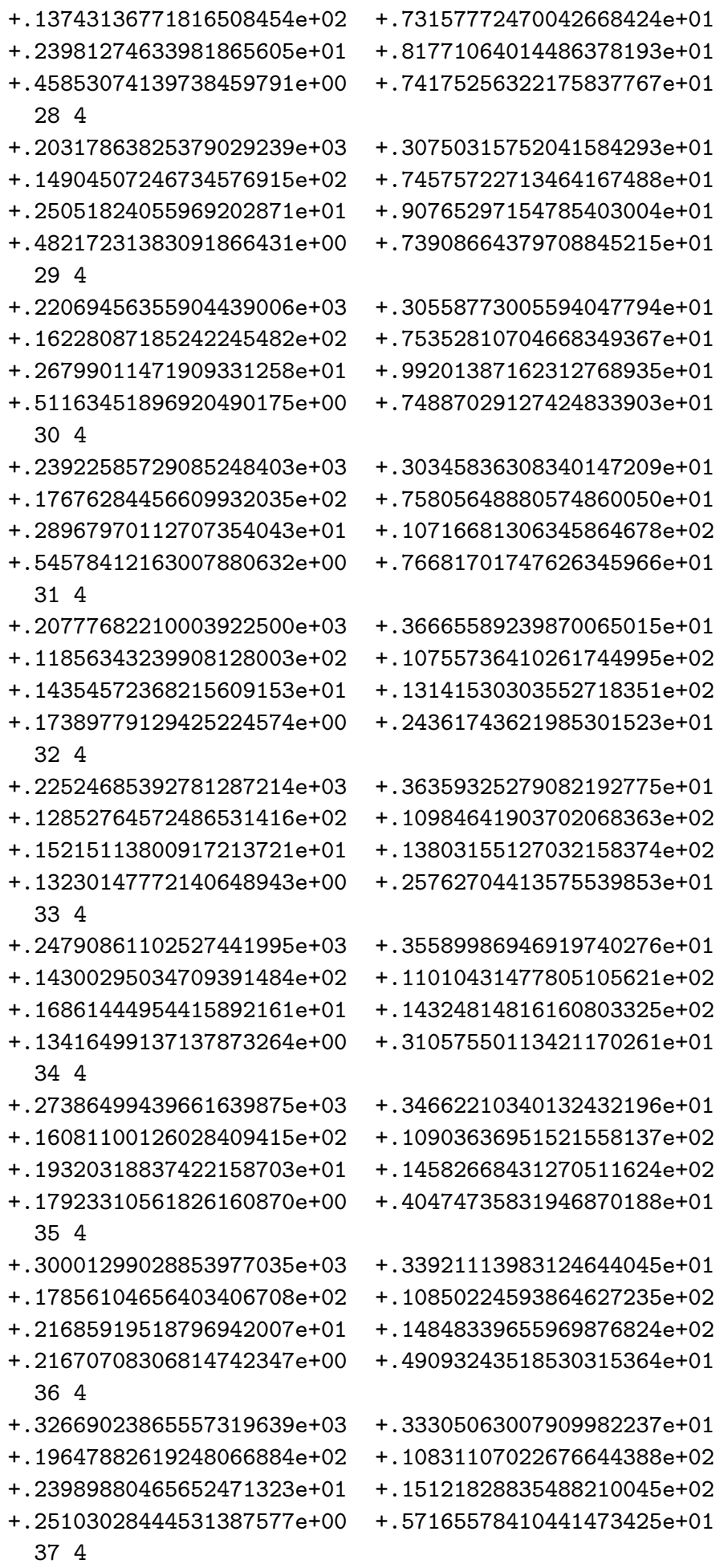




\begin{tabular}{|c|c|}
\hline$+.37163737826238113430 \mathrm{e}+03$ & $+.31466666484776166178 \mathrm{e}+01$ \\
\hline$+.23248544533094692908 \mathrm{e}+02$ & $+.10341351681057330980 e+02$ \\
\hline$+.28726598551454175825 e+01$ & $+.15563074945782764186 e+02$ \\
\hline $\begin{aligned}+. & 27773911546919278777 \mathrm{e}+00 \\
& 384\end{aligned}$ & $+.69489067246822882167 e+01$ \\
\hline$+.37072341161894166681 \mathrm{e}+03$ & $+.33133131598951131789 \mathrm{e}+01$ \\
\hline$+.22369820917706722631 \mathrm{e}+02$ & $+.11069248049555791375 e+02$ \\
\hline$+.27431178263461401512 \mathrm{e}+01$ & $+.15751396571059824070 \mathrm{e}+02$ \\
\hline $\begin{array}{l}+.22670958449869717963 e+00 \\
\quad 394\end{array}$ & $+.68660422194892713765 \mathrm{e}+01$ \\
\hline$+.38194339180832728613 e+03$ & $+.33897687777580409061 \mathrm{e}+01$ \\
\hline$+.22657005971801517501 \mathrm{e}+02$ & $+.11515238880840111505 \mathrm{e}+02$ \\
\hline$+.27818156103527597502 \mathrm{e}+01$ & $+.15744363112944809796 \mathrm{e}+02$ \\
\hline $\begin{array}{l}+.23142082707081156801 \mathrm{e}+00 \\
\quad 404\end{array}$ & $+.73506292284570377920 \mathrm{e}+01$ \\
\hline$+.40412975489707895010 \mathrm{e}+03$ & $+.33912387012959417039 e+01$ \\
\hline$+.23913120490512053721 \mathrm{e}+02$ & $+.11698493461811265601 e+02$ \\
\hline$+.29281223941744981287 \mathrm{e}+01$ & $+.15897124348300313556 \mathrm{e}+02$ \\
\hline $\begin{array}{l}+.25096601775828317782 \mathrm{e}+00 \\
\quad 414\end{array}$ & $+.80131434885924791388 e+01$ \\
\hline$+.43200075322658102319 \mathrm{e}+03$ & $+.33619764754289541514 \mathrm{e}+01$ \\
\hline$+.25649609677505364113 e+02$ & $+.11759141278005034150 \mathrm{e}+02$ \\
\hline$+.31272636914975559143 e+01$ & $+.16141877715462901120 \mathrm{e}+02$ \\
\hline $\begin{array}{l}+.27614878489011475330 \mathrm{e}+00 \\
\quad 424\end{array}$ & $+.87370045311031105790 e+01$ \\
\hline$+.46268918558642525532 \mathrm{e}+03$ & $+.33241931072269496919 e+01$ \\
\hline$+.27620091978879956212 \mathrm{e}+02$ & $+.11775004054054392252 \mathrm{e}+02$ \\
\hline$+.33502415418868519603 e+01$ & $+.16421219554119644948 e+02$ \\
\hline $\begin{array}{l}+.30463722353499470739 e+00 \\
\quad 434\end{array}$ & $+.94795832845990131073 e+01$ \\
\hline$+.49504830506721169066 \mathrm{e}+03$ & $+.32859988207200458037 e+01$ \\
\hline$+.29724523813339572593 e+02$ & $+.11777513760113202247 e+02$ \\
\hline$+.35840719287190991983 e+01$ & $+.16713996790559294240 \mathrm{e}+02$ \\
\hline $\begin{array}{l}+.33542029325897034058 \mathrm{e}+00 \\
\quad 444\end{array}$ & $+.10222490628607457709 \mathrm{e}+02$ \\
\hline$+.50788917516825208035 e+03$ & $+.33568004375740026291 e+01$ \\
\hline$+.30119638007448954451 \mathrm{e}+02$ & $+.12183651199011928789 e+02$ \\
\hline$+.35989334168125757504 \mathrm{e}+01$ & $+.16732947240496758997 e+02$ \\
\hline $\begin{array}{l}+.36368976141299972161 e+00 \\
\quad 454\end{array}$ & $+.10726601122917309585 e+02$ \\
\hline$+.52496383750584292732 \mathrm{e}+03$ & $+.34042696795165551855 \mathrm{e}+01$ \\
\hline$+.30919224799048707681 \mathrm{e}+02$ & $+.12498923939454255853 e+02$ \\
\hline$+.36440391113842645119 \mathrm{e}+01$ & $+.16870466086010282043 e+02$ \\
\hline $\begin{array}{l}+.39297773558866046385 \mathrm{e}+00 \\
\quad 464\end{array}$ & $+.11226340295018906918 \mathrm{e}+02$ \\
\hline$+.54537148473898028396 \mathrm{e}+03$ & $+.34352063845776484904 e+01$ \\
\hline$+.32017351723397207779 \mathrm{e}+02$ & $+.12743509881914651382 \mathrm{e}+02$ \\
\hline$+.37145429669883926110 \mathrm{e}+01$ & $+.17096800752115824492 e+02$ \\
\hline$+.42339611975503888333 e+00$ & $+.11724482981391875635 \mathrm{e}+02$ \\
\hline
\end{tabular}


474

$+.56848334028080858164 \mathrm{e}+03$

$+.33345924933420031056 \mathrm{e}+02$

$+.34544421984266276586 \mathrm{e}+01$

$+.38059246866006447837 \mathrm{e}+01$

$+.12934294037833886018 \mathrm{e}+02$

$+.17389101155187288809 \mathrm{e}+02$

$+.45499468116272807546 \mathrm{e}+00$

$+.12222162608552197514 \mathrm{e}+02$

484

$+.59388588982128796181 \mathrm{e}+03$

$+.34652635118576361543 \mathrm{e}+01$

$+.34862597588803264317 \mathrm{e}+02$

$+.13083885898252915897 \mathrm{e}+02$

$+.39146777891016737707 e+01$

$+.17730959451884514142 \mathrm{e}+02$

$+.48779609479059794305 \mathrm{e}+00$

$+.12719891138004933806 \mathrm{e}+02$ 495

$+.74504533341674428055 \mathrm{e}+03$

$+.47589716263493248893 \mathrm{e}+02$

$+.29874634939188884888 \mathrm{e}+01$

$+.11154282804456501447 \mathrm{e}+02$

$+.59712038282804846794 \mathrm{e}+01$

$+.17314739405885692879 \mathrm{e}+02$

$+.74300422091938889735 \mathrm{e}+00$

$+.14971163078231132275 \mathrm{e}+02$

$+.84660995560378415338 \mathrm{e}-01$

$+.15723512175077849100 \mathrm{e}+01$ 505

$+.79702852654173314139 \mathrm{e}+03$

$+.51533843511592459550 \mathrm{e}+02$

$+.29316341933211216742 \mathrm{e}+01$

$+.10995396237542593334 \mathrm{e}+02$

$+.65092596715707926475 \mathrm{e}+01$

$+.17451448107477579837 \mathrm{e}+02$

$+.80544055332868458826 e+00$

$+.15716102363338380092 \mathrm{e}+02$

$+.66326146535977302557 \mathrm{e}-01$

$+.19054190983203250623 \mathrm{e}+01$ 515

$+.85433263573360495731 \mathrm{e}+03$

$+.28687229851761811778 \mathrm{e}+01$

$+.56030106441107747302 e+02$

$+.71434023126589638652 \mathrm{e}+01$

$+.10796297417605338766 \mathrm{e}+02$

$+.17532917379237792734 \mathrm{e}+02$

$+.88347126319429414394 \mathrm{e}+00$

$+.16400893313073775167 \mathrm{e}+02$

$+.67335798553194526531 \mathrm{e}-01$ 525

$+.91216680013767399628 \mathrm{e}+03$

$+.60593809682671006622 \mathrm{e}+02$

$+.24011689049069121549 \mathrm{e}+01$

$+.28147490944765846995 \mathrm{e}+01$

$+.10621553493355722879 \mathrm{e}+02$

$+.17535679854859787483 \mathrm{e}+02$

$+.78128697037188814937 \mathrm{e}+01$

$+.16749961304308713660 \mathrm{e}+02$

$+.98647849331351539671 \mathrm{e}+00$

$+.32780562529991912778 \mathrm{e}+01$

535

$+.97123265740465906255 \mathrm{e}+03+.27669360798826062817 \mathrm{e}+01$

$+.65252543199773385315 \mathrm{e}+02+.10468708135547245616 \mathrm{e}+02$

$+.84954736819143606181 \mathrm{e}+01+.17546759842438655658 \mathrm{e}+02$

$+.10874117592443088735 \mathrm{e}+01+.17126418429611765221 \mathrm{e}+02$

$+.12699302114117239117 \mathrm{e}+00+.40911775125197272236 \mathrm{e}+01$ 545

$+.10318412270302938313 e+04$

$+.70039902307632663117 \mathrm{e}+02$

$+.27237667219329389421 \mathrm{e}+01$

$+.10330894101872670405 \mathrm{e}+02$

$+.91969976448947273582 \mathrm{e}+01+.17563256036021080262 \mathrm{e}+02$

$+.11869856131289064870 \mathrm{e}+01+.17530860453395113778 \mathrm{e}+02$

$+.14857777514764354720 \mathrm{e}+00+.48512226867781966123 \mathrm{e}+01$ 555

$+.11493826345739148054 \mathrm{e}+04+.25533576720595384602 \mathrm{e}+01$

$+.82074003023619112491 \mathrm{e}+02+.95665281654822195899 \mathrm{e}+01$ 


\begin{tabular}{|c|c|}
\hline $.11211986732106572843 e$ & $+.17061280408746025814 \mathrm{e}+02$ \\
\hline $.15279462333396209482 e+01$ & $+.17732211210674003336 \mathrm{e}+02$ \\
\hline $\begin{array}{l}20092255135604541527 e+00 \\
565\end{array}$ & $+.70866225430382127994 e+01$ \\
\hline $.11696947757016588526 \mathrm{e}+04$ & $+.26213312447246266653 e+01$ \\
\hline $97198378601 e+02$ & +.9917126222580187 \\
\hline $6 e+02$ & +.1730860069579679 \\
\hline $3 e+01$ & $+.18136517413864287698 e+02$ \\
\hline $\begin{array}{l}.17008617153559455691 e+00 \\
575\end{array}$ & $+.70164244230341081479 e+01$ \\
\hline $0 e+04$ & +.261955613751081661 \\
\hline $8 e+02$ & $2 e+01$ \\
\hline $3 e+02$ & +.174528 \\
\hline $1 e+01$ & +.184265 \\
\hline $\begin{array}{l}+.17220089046882704 \\
\quad 585\end{array}$ & +.754332022506404031 \\
\hline $6 e+04$ & +.2627 \\
\hline $3 e+02$ & +.100 \\
\hline $\mathrm{e}+02$ & $05 e+02$ \\
\hline+.160717 & +.18927 \\
\hline $\begin{array}{l}+.1807949 \\
\quad 595\end{array}$ & +.7675 \\
\hline $32 e+04$ & +.263808 \\
\hline $2 e+02$ & +.1011 \\
\hline+.121071 & +.179 \\
\hline+.1653781 & $085116 e+02$ \\
\hline $\begin{array}{c}+.188372 \\
605\end{array}$ & $8 e+01$ \\
\hline $6 e+04$ & +.2650605015987261 \\
\hline $1 e+02$ & +.101 \\
\hline+.1238892 & +.182 \\
\hline+.170240 & $4 e+02$ \\
\hline $\begin{array}{l}+.1952797675 \\
\quad 615\end{array}$ & $14 e+01$ \\
\hline+.14232939068289442 & +.266494 \\
\hline$+.97328657834613684470 e+02$ & +.1027445907168 \\
\hline+.12661 & $e+02$ \\
\hline+.17523 & $e+02$ \\
\hline $\begin{array}{l}+.2016862438051 \\
\quad 625\end{array}$ & $55 e+01$ \\
\hline$+.14745487132299602158 e+04$ & +.26807923239534 \\
\hline$+.10024430189239065721 e+03$ & $+.10356881026567634681 e+02$ \\
\hline$+.12925522095087921053 e+02$ & $9417404521530458 e+02$ \\
\hline+.180347270188 & $228382 e+02$ \\
\hline $\begin{aligned}+ & 2076935812660 \\
& 635\end{aligned}$ & $+.78689320030151853081 \mathrm{e}+01$ \\
\hline$+.15264150536618301112 \mathrm{e}+04$ & $+.26978640905721766211 \mathrm{e}+01$ \\
\hline$+.10314004849790670614 e+03$ & $+.10439868003977639808 e+02$ \\
\hline$+.13181653182291660523 e+02$ & $+.19087352645971370073 e+02$ \\
\hline
\end{tabular}


$+.18554646230381475835 \mathrm{e}+01+.21897912345772990911 \mathrm{e}+02$

$+.21336744705076069654 \mathrm{e}+00+.78770029137058225877 \mathrm{e}+01$ 645

$+.15789784735126056335 \mathrm{e}+04+.27159305699306148774 \mathrm{e}+01$

$+.10602501158566269475 \mathrm{e}+03+.10522796214675617561 \mathrm{e}+02$

$+.13431158961640053435 \mathrm{e}+02+.19387452129770386514 \mathrm{e}+02$

$+.19082409938489719327 \mathrm{e}+01+.22497378964585917405 \mathrm{e}+02$

$+.21875121147602400890 \mathrm{e}+00+.78764421210374636418 \mathrm{e}+01$ 655

$+.16279257815186742923 e+04+.27411226932956958183 e+01$

$+.10858046918725347295 \mathrm{e}+03+.10616350835073855907 \mathrm{e}+02$

$+.13712227614246241933 \mathrm{e}+02+.19538239265378358275 \mathrm{e}+02$

$+.19934065152409421501 \mathrm{e}+01+.23132099102842867011 \mathrm{e}+02$

$+.22607538133284423461 \mathrm{e}+00+.79721881034092229887 \mathrm{e}+01$ 665

$+.16757366368652999276 \mathrm{e}+04+.27697835225437479078 \mathrm{e}+01$

$+.11092891605390537645 \mathrm{e}+03+.10722503380066446967 \mathrm{e}+02$

$+.13944492993755349266 \mathrm{e}+02+.19736802312959688194 \mathrm{e}+02$

$+.20713613685444629290 \mathrm{e}+01+.23730486350905376132 \mathrm{e}+02$

$+.23293090457243363435 \mathrm{e}+00+.80404244335247407995 \mathrm{e}+01$ 675

$+.17224510232360898468 \mathrm{e}+04$

$+.11309508331285164387 \mathrm{e}+03$

$+.28015830284582851640 \mathrm{e}+01$

$+.10838293069461611601 \mathrm{e}+02$

$+.19978990892678130435 \mathrm{e}+02$

$+.14135448633292121789 \mathrm{e}+02$

$+.24293655068020399645 \mathrm{e}+02$

$+.21434468833925621800 \mathrm{e}+0$

$+.80874779413815731556 e+01$

685

$+.17681273694341087398 \mathrm{e}+04+.28361728534561255897 \mathrm{e}+01$

$+.11510451705123839658 \mathrm{e}+03+.10961108480750576628 \mathrm{e}+02$

$+.14292436046614584054 \mathrm{e}+02+.20261631825154297223 \mathrm{e}+02$

$+.22106447962734520737 \mathrm{e}+01+.24823569072814496512 \mathrm{e}+02$

$+.24552713219420616199 e+00+.81175177678245040477 e+01$ 695

$+.18128387097425374031 \mathrm{e}+04+.28732306358723144930 \mathrm{e}+01$

$+.11698049742273002375 \mathrm{e}+03+.11088803657353719962 \mathrm{e}+02$

$+.14422182620212654739 \mathrm{e}+02+.20582107554913643492 \mathrm{e}+02$

$+.22736936308261366496 \mathrm{e}+01+.25322405700084024151 \mathrm{e}+02$

$+.25134177827762767419 \mathrm{e}+00+.81334524517762979017 \mathrm{e}+01$ 705

$+.18566685254316594285 e+04+.29124652371565373783 e+01$

$+.11874345107133860150 \mathrm{e}+03+.11219670947580419406 \mathrm{e}+02$

$+.14530686065898029811 \mathrm{e}+02+.20938069255347597683 \mathrm{e}+02$

$+.23331738881401942761 e+01+.25792350278740494423 e+02$

$+.25686096532697333606 \mathrm{e}+00+.81374442811749511096 \mathrm{e}+01$ 715

$+.18997375959050908652 \mathrm{e}+04+.29535955441201374457 \mathrm{e}+01$

$+.12041203401518791747 \mathrm{e}+03+.11352349978687230449 \mathrm{e}+02$

$+.14623290920474742214 \mathrm{e}+02+.21327243843421123933 \mathrm{e}+02$

$+.23895731400544327792 \mathrm{e}+01+.26235577299844828812 \mathrm{e}+02$ 


$\begin{array}{ll}+.26209875138329045085 \mathrm{e}+00 & +.81312333339266793605 \mathrm{e}+01 \\ +725 & \\ +.19673161513254125348 \mathrm{e}+04 & +.29621161002957168217 \mathrm{e}+01 \\ +.12434022381142522564 \mathrm{e}+03 & +.11361133113996146592 \mathrm{e}+02 \\ +.15073083794221049984 \mathrm{e}+02 & +.21578072665372014527 \mathrm{e}+02 \\ +.24808185704179228484 \mathrm{e}+01 & +.26565925422554728476 \mathrm{e}+02 \\ +.26617131686220868124 \mathrm{e}+00 & +.85327526977813935829 \mathrm{e}+01 \\ +735 & \\ +.20700813765288223264 \mathrm{e}+04 & +.29277615621936452850 \mathrm{e}+01 \\ +.13149175287923276758 \mathrm{e}+03 & +.11205512222519954671 \mathrm{e}+02 \\ +.15991001325343890074 \mathrm{e}+02 & +.21617220829977906263 \mathrm{e}+02 \\ +.26318773077354023292 \mathrm{e}+01 & +.27084534015420166982 \mathrm{e}+02 \\ +.28043101980165810474 \mathrm{e}+00 & +.91649713698883267988 \mathrm{e}+01 \\ +745 & \\ +.21895493581497282395 \mathrm{e}+04 & +.28789204905262306730 \mathrm{e}+01 \\ +.14025127395857804340 \mathrm{e}+03 & +.10991065963882566690 \mathrm{e}+02 \\ +.17133447569749620252 \mathrm{e}+02 & +.21575368819978679375 \mathrm{e}+02 \\ +.28098978707573803615 \mathrm{e}+01 & +.27659569431312725045 \mathrm{e}+02 \\ +.26479423678868878637 \mathrm{e}+04 & +.28690702858987777134 \mathrm{e}+01 \\ +.16965288496596832204 \mathrm{e}+03 & +.10736842366501742438 \mathrm{e}+02 \\ +.20849001035167128167 \mathrm{e}+02 & +.22231851688015446299 \mathrm{e}+02 \\ +.34909991955041559319 \mathrm{e}+01 & +.28774342144019749205 \mathrm{e}+02 \\ +.42063212480622336363 \mathrm{e}+00 & +.13387893515564284345 \mathrm{e}+02 \\ +755 & \end{array}$


805

$+.27479281346759772134 \mathrm{e}+04$

$+.17599878082315510778 \mathrm{e}+03$

$+.21649674830696111463 \mathrm{e}+02$

$+.36289678032455827459 \mathrm{e}+01$

$+.44820670782959366692 \mathrm{e}+00$ 816

$+.31644430017902674779 \mathrm{e}+04$

$+.21572530656955273349 \mathrm{e}+03$

$+.27161342191636469259 \mathrm{e}+02$

$+.44914277801281687709 \mathrm{e}+01$

$+.60044016507879410021 \mathrm{e}+00$

$+.68530932050186837842 \mathrm{e}-01$ 826

$+.33326463110290519887 \mathrm{e}+04$

$+.22900310905632420178 \mathrm{e}+03$

$+.28975969438335592188 \mathrm{e}+02$

$+.48105585257086867361 \mathrm{e}+01$

$+.64280898159203082927 \mathrm{e}+00$

$+.47924330717300866072 \mathrm{e}-01$ 836

$+.35122165088553368970 \mathrm{e}+04$

$+.24339214593688772585 \mathrm{e}+03$

$+.30978532465816319835 \mathrm{e}+02$

$+.51724235894520245522 \mathrm{e}+01$

$+.69729147895113773667 \mathrm{e}+00$

$+.46483440633541432225 \mathrm{e}-01$ 846

$+.36875639473458099862 e+04$

$+.25699663742660035012 \mathrm{e}+03$

$+.32885250410274436152 \mathrm{e}+02$

$+.55372807516587905839 \mathrm{e}+01$

$+.76695240190109891076 \mathrm{e}+00$

$+.77909417059715903711 \mathrm{e}-01$ 856

$+.38672700297383376974 \mathrm{e}+04$

$+.27079708409449999366 \mathrm{e}+03$

$+.34834221835135341239 \mathrm{e}+02$

$+.59163326065550116963 \mathrm{e}+01$

$+.83714257744570818238 \mathrm{e}+00$

$+.10024708223935701640 \mathrm{e}+00$ 866

$+.40511370697500908635 e+04$

$+.28477593787039301706 \mathrm{e}+03$

$+.36824980911702692865 \mathrm{e}+02$

$+.63096008469553255530 \mathrm{e}+01$

$+.90780562491788329230 \mathrm{e}+00$

$+.11834115297115231510 \mathrm{e}+00$ 876
$+.28687766249959008053 e+01$

$+.10687584444214812912 \mathrm{e}+02$

$+.22362991007380984298 \mathrm{e}+02$

$+.28981078296242676723 \mathrm{e}+02$

$+.14099569627165625262 \mathrm{e}+02$

$+.25898355236158902471 \mathrm{e}+01$

$+.96246431246896855497 \mathrm{e}+01$

$+.20809716003886912456 \mathrm{e}+02$

$+.29952533588261982205 \mathrm{e}+02$

$+.15700427135109986694 \mathrm{e}+02$

$+.13228446244355428485 \mathrm{e}+01$

$+.25510270843967342072 \mathrm{e}+01$

$+.94477355440436975334 \mathrm{e}+01$

$+.20605202222691935705 \mathrm{e}+02$

$+.30226868107463972659 \mathrm{e}+02$

$+.16563554283376984155 \mathrm{e}+02$

$+.16056127580266757405 \mathrm{e}+01$

$+.25107682966586122455 \mathrm{e}+01$

$+.92637277362678042288 \mathrm{e}+01$

$+.20354334911567376745 \mathrm{e}+02$

$+.30433822127772071943 e+02$

$+.17386456246734130171 \mathrm{e}+02$

$+.20508906810000046669 \mathrm{e}+01$

$+.24806720777508070354 \mathrm{e}+01$

$+.91185410850077186967 \mathrm{e}+01$

$+.20141169412875392928 \mathrm{e}+02$

$+.30533455818589890446 \mathrm{e}+02$

$+.17863120770146898394 e+02$

$+.28630408356292925010 \mathrm{e}+01$

$+.24538110315323277185 \mathrm{e}+01$

$+.89854236795571566622 \mathrm{e}+01$

$+.19932186171355124066 \mathrm{e}+02$

$+.30613532620204298900 \mathrm{e}+02$

$+.18373965488949320224 \mathrm{e}+02$

$+.36410810084017724287 e+01$

$+.24300095420946064498 \mathrm{e}+01$

$+.88632447318808189517 \mathrm{e}+01$

$+.19727281480571014606 \mathrm{e}+02$

$+.30677132568876660600 \mathrm{e}+02$

$+.18918099140596643612 \mathrm{e}+02$

$+.43842325359802557809 e+01$ 
$+.43539847943817889650 \mathrm{e}+04+.23429209770104878628 \mathrm{e}+01$

$+.31502218975440361631 \mathrm{e}+03+.84217316762871401933 \mathrm{e}+01$

$+.41780895975171042604 \mathrm{e}+02+.18761468929404893311 \mathrm{e}+02$

$+.73246351832093548642 e+01+.30084189577167919949 e+02$

$+.12167680803927787948 \mathrm{e}+01+.18988459624861476385 \mathrm{e}+02$

$+.18531646855260040879 e+00+.74012292152680822992 e+01$ 886

$+.44483506218660839615 e+04+.23809454644266776589 e+01$

$+.31637235237572965497 e+03+.85759331031683028743 e+01$

$+.41599655197053687089 e+02+.19127747791660405618 e+02$

$+.73320030188586031866 \mathrm{e}+01+.30322680706875483984 \mathrm{e}+02$

$+.11859426306615156698 \mathrm{e}+01+.19296658373611664160 \mathrm{e}+02$

$+.16058344554826791978 \mathrm{e}+00+.72960345602574657047 \mathrm{e}+01$ 896

$+.46031531564153656986 \mathrm{e}+04+.23903650383882533962 \mathrm{e}+01$

$+.32430071106692241723 e+03+.86119611299271118442 e+01$

$+.42437246625992947596 \mathrm{e}+02+.19293262342063462455 \mathrm{e}+02$

$+.74892450697690965890 e+01+.30584541746206084794 e+02$

$+.11874081908615181964 \mathrm{e}+01+.19606439682466565325 \mathrm{e}+02$

$+.15164436711332391191 e+00+.75134300609485221858 e+01$ 906

$+.47971151030446662116 e+04+.23795898439698880478 e+01$

$+.33826880192816451082 \mathrm{e}+03+.85331468200544571276 \mathrm{e}+01$

$+.44295335407383541629 e+02+.19245716363499046028 e+02$

$+.78319083873759350076 \mathrm{e}+01+.30679118109521893171 \mathrm{e}+02$

$+.12477414312105594584 \mathrm{e}+01+.20130670917808011706 \mathrm{e}+02$

$+.16327011497437024786 e+00+.80317579451467039196 e+01$ 916

$+.49883843473347966388 \mathrm{e}+04+.23753063722205039939 \mathrm{e}+01$

$+.35079261962815327693 e+03+.84911083041889169853 e+01$

$+.45860626299108343141 e+02+.19275177917826605551 e+02$

$+.81275157307497156059 \mathrm{e}+01+.30760434782802710068 \mathrm{e}+02$

$+.12948293491755238100 \mathrm{e}+01+.20707670300884711543 \mathrm{e}+02$

$+.17433619558418046722 \mathrm{e}+00+.83903023220765518582 \mathrm{e}+01$ 926

$+.51830661629723292921 e+04+.23735771858370988371 e+01$

$+.36286617208313892515 \mathrm{e}+03+.84648705410982652496 \mathrm{e}+01$

$+.47296628009567850930 \mathrm{e}+02+.19347923356133982310 \mathrm{e}+02$

$+.83931120859276319413 e+01+.30855948029935142422 e+02$

$+.13330953102037347731 e+01+.21320965705131993097 e+02$

$+.18401729827822357817 e+00+.86367151818635180847 e+01$ 936

$+.53845435728636195778 e+04+.23728653367085984718 e+01$

$+.37492172838433873261 e+03+.84456432596417946307 e+01$

$+.48682558935056744437 e+02+.19443484432954414085 e+02$

$+.86430481254931232728 e+01+.30959928495385360443 e+02$

$+.13667738267877879989 e+01+.21974025687078247004 e+02$

$+.19260759242970482328 e+00+.88040527882315853653 e+01$ 946 


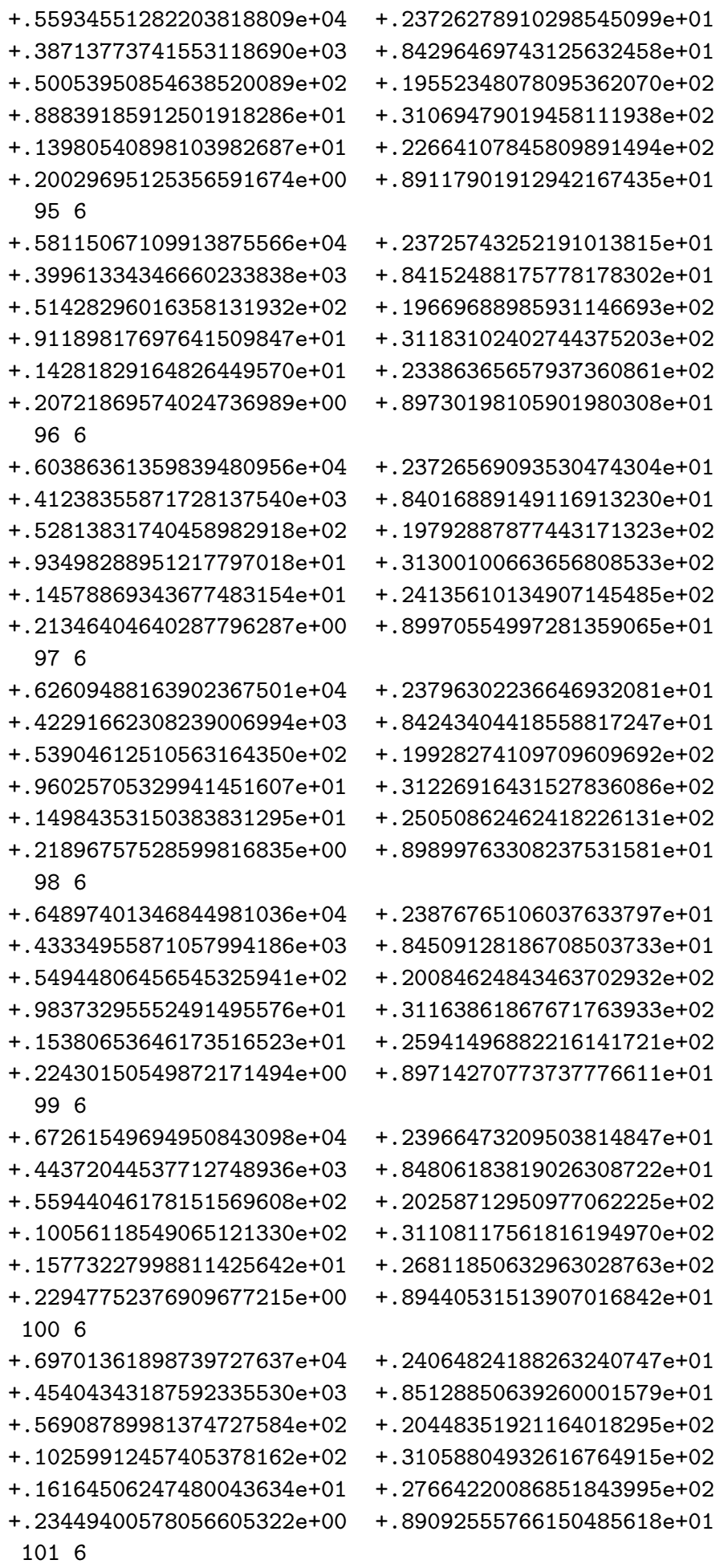


$+.72231442425609014323 e+04+.24170541416903781674 \mathrm{e}+01$

$+.46434828194783742001 \mathrm{e}+03+.85472803929013371146 \mathrm{e}+01$

$+.57845026820999606173 \mathrm{e}+02+.20651830520976053376 \mathrm{e}+02$

$+.10449227089089557521 \mathrm{e}+02+.31016261177045224748 \mathrm{e}+02$

$+.16555508524664383349 \mathrm{e}+01+.28499613539628402704 \mathrm{e}+02$

$+.23935006172299832579 \mathrm{e}+00+.88679602277586038902 \mathrm{e}+01$

1026

$+.74856133966236282386 \mathrm{e}+04+.24282610034738251006 \mathrm{e}+01$

$+.47466477442887573459 \mathrm{e}+03+.85832214235225030873 \mathrm{e}+01$

$+.58760145327839364080 \mathrm{e}+02+.20867214864905726500 \mathrm{e}+02$

$+.10624698106362063148 \mathrm{e}+02+.30981660197343698257 \mathrm{e}+02$

$+.16946873774494313422 \mathrm{e}+01+.29318647910565503535 \mathrm{e}+02$

$+.24404577984956426995 \mathrm{e}+00+.88209946001887435202 \mathrm{e}+01$ 